\title{
Integrating Small RNA Sequencing with QTL Mapping for Identification of miRNAs and Their Target Genes Associated with Heat Tolerance at the Flowering Stage in Rice
}

\author{
Qing Liu 1,2†, Tifeng Yang ${ }^{1,2 \dagger}$, Ting $\mathrm{Yu}^{3+}$, Shaohong Zhang ${ }^{1,2}$, Xingxue Mao ${ }^{1,2}$, \\ Junliang Zhao ${ }^{1,2}$, Xiaofei Wang ${ }^{1,2}$, Jingfang Dong ${ }^{1,2}$ and Bin Liu ${ }^{1,2 *}$ \\ ' Guangdong Key Laboratory of New Technology in Rice Breeding, Guangzhou, China, ${ }^{2}$ Rice Research Institute, Guangdong \\ Academy of Agricultural Sciences, Guangzhou, China, ${ }^{3}$ Agro-biological Gene Research Center, Guangdong Academy of \\ Agricultural Sciences, Guangzhou, China
}

OPEN ACCESS

Edited by:

Vicent Arbona,

Jaume I University, Spain

Reviewed by:

Yao-Cheng Lin,

Academia Sinica, Taiwan

Jun Yan,

Purdue University, USA

Xin-Xiang Peng,

South China Agricultural University,

China

*Correspondence:

Bin Liu

Ibgz1009@163.com

${ }^{\dagger}$ These authors have contributed equally to this work.

Specialty section:

This article was submitted to

Plant Abiotic Stress,

a section of the journal

Frontiers in Plant Science

Received: 20 November 2016 Accepted: 09 January 2017

Published: 24 January 2017

Citation: Liu Q, Yang T, Yu T, Zhang S, Mao X Zhao J, Wang $X$, Dong J and Liu B (2017) Integrating Small RNA Sequencing with QTL Mapping for Identification of miRNAs and Their Target Genes Associated with Heat Tolerance at the Flowering Stage in

Rice. Front. Plant Sci. 8:43. doi: 10.3389/fp/s.2017.00043
Although, microRNAs (miRNAs) have been reported to be associated with heat tolerance at the seedling stage in rice, their involvement in heat tolerance at the flowering stage is still unknown. In this study, small RNA profiling was conducted in a heat-tolerant variety Gan-Xiang-Nuo (GXN) and a heat-sensitive variety Hua-Jing-Xian-74 (HJX), respectively. Totally, 102 miRNAs were differentially expressed (DE) under heat stress. Compared to HJX, GXN had more DE miRNAs and its DE miRNAs changed earlier under heat stress. Plant Ontology (PO) analysis of the target genes revealed that many DE miRNAs were involved in flower development. As a parallel experiment, QTL mapping was also conducted and four QTLs for heat tolerance at the flowering stage were identified using chromosome single-segment substitution lines derived from GXN and HJX. Further, through integrating analysis of DE miRNAs with QTLs, we identified 8 target genes corresponding to 26 miRNAs within the four QTL regions. Some meaningful target genes such as LOC_Os12g42400, SGT1, and pectinesterase were within the QTL regions. The negative correlation between miR169r-5p and its target gene LOC_Os12g42400 was confirmed under heat stress, and overexpression of miR169r-5p enhanced heat tolerance at flowering stage in rice. Our results demonstrate that the integrated analysis of genome-wide miRNA profiling with QTL mapping can facilitate identification of miRNAs and their target genes associated with the target traits and the limited candidates identified in this study offer an important source for further functional analysis and molecular breeding for heat tolerance in rice.

Keywords: miRNA, heat stress, RNA Sequencing, QTL mapping, rice

\section{INTRODUCTION}

Rice is the major food for over half of the world population. Therefore, sustainable rice production is very important for the world food security. However, rice farming is always subjected to different abiotic and biotic stresses. Heat stress is one of the major abiotic stresses that significantly affect rice growth and development. The previous study showed that a $7 \sim 8 \%$ yield decrease in rice for 
each $1^{\circ} \mathrm{C}$ increase in daytime maximum/nighttime minimum temperature from $28 / 21$ to $34 / 27^{\circ} \mathrm{C}$ (Baker et al., 1992). Flowering (anthesis and fertilization) is the most sensitive stage to temperature in rice (Satake and Yoshida, 1978; Farrell et al., 2006). Rice plants at anthesis that are exposed to temperatures $>35^{\circ} \mathrm{C}$ for 5 days are sterile and set no seed (Satake and Yoshida, 1978). Exposure to $33.7^{\circ} \mathrm{C}$ for $1 \mathrm{~h}$ at flowering stage could cause IR64 (lowland indica) and Azucena (upland japonica) significant sterility (Jagadish et al., 2007). It is noteworthy that global warming has exerted a significant effect on rice production. A study conducted by International Rice Research Institute (IRRI) revealed that annual mean maximum and minimum temperatures increased by 0.35 and $1.13^{\circ} \mathrm{C}$, respectively, for the period of 1979-2003 at International Rice Research Institute, Manila, Philippines, and grain yields declined by $10 \%$ for each $1^{\circ} \mathrm{C}$ increase in growing-season minimum temperature in the dry season (Peng et al., 2004). Furthermore, a report from the Intergovernmental Panel on Climatic Change (IPCC) predicted that plants would be grown in much warmer environments with the average surface temperature increase of $2.0 \sim 4.5^{\circ} \mathrm{C}$ by the end of this century (IPCC, 2007). Therefore, it is an urgent task to develop rice variety with heat tolerance to cope with the world climate change. However, heat tolerance in rice is a complex trait controlled by multiple genes (QTLs; Tabata et al., 2007; Ye et al., 2012). Understanding the mechanisms of rice in response to heat stress and identifying the genes associated with heat tolerance are the prerequisite for effective molecular breeding for heat tolerance in rice.

Recently, more and more evidences indicated that microRNAs (miRNAs), a sort of endogenous small non-coding RNAs, play pivotal roles in plant stress responses at the post-transcriptional level (Liu and Chen, 2010). miRNAs exert their functions through negatively modulating the expression of genes by promoting the degradation of target mRNAs or by inhibiting translation. A large number of miRNAs have been demonstrated to function in regulation of plant response to biotic and abiotic stresses, including pathogen invasion (Sunkar et al., 2006; Boccara et al., 2014; Li Y. et al., 2014), nutrient starvation (Fujii et al., 2005; Kawashima et al., 2009, 2011; He et al., 2014), cold (Sunkar and Zhu, 2004; Lv et al., 2010), salt (Song et al., 2013; Zhou et al., 2013), drought (Ni et al., 2013; Song et al., 2013; Fang et al., 2014), and oxidative stresses (Sunkar et al., 2006; Jagadeeswaran et al., 2009). For example, miR319 positively regulates plant response to drought and salinity stress (Zhou et al., 2013). MiR394 negatively regulates salt tolerance but positively regulates drought tolerance in an abscisic acid-dependent manner in Arabidopsis (Song et al., 2013). Furthermore, miR826 and miR5090 mediate nitrogen starvation adaptation via regulation of glucosinolate synthesis in Arabidopsis (He et al., 2014).

With the rapid development of high throughput sequencing technology in the last few years, a genome-wide search for miRNAs that are responsible for heat stress has been reported in many plants such as wheat (Xin et al., 2010), barley (Kruszka et al., 2014), Chinese cabbage (Yu et al., 2011), Chinese white poplar (Chen et al., 2012), Arabidopsis (Guan et al., 2013; Stief et al., 2014), celery (Li M. Y. et al., 2014), and rice (Jeong et al., 2011). Although, the study of miRNAs in heat tolerance has been made in rice at the seedling stage, a genome-wide identification of miRNAs responsive to heat stress at the flowering stage, the most sensitive stage to temperature and important to grain yield in rice has not been conducted. According to the previous study, many miRNAs express differently in different tissues in rice (Jeong et al., 2011). We reason that there might be different sets of miRNAs in response to heat stress at the seedling stage and the flowering stage in rice, respectively. Thus, to fully understand the mechanism of rice in response to heat stress and identify the potential functional genes useful for molecular breeding, it is worthy to perform a genome-wide profiling of miRNAs at the flowering stage in rice.

Previous genome-wide miRNA profiling experiments have been done using only one genotype. In this study, to better understand the mechanism of miRNA in regulation of heat tolerance in rice, two parallel small RNA profiling experiments were performed at the flowering stage in a heat-tolerant variety Gan-Xiang-Nuo (GXN) and a heat-sensitive variety HuaJing-Xian-74 (HJX). We identified the conserved miRNAs in GXN and HJX, respectively. More differentially expressed (DE) miRNAs and earlier expression change of the DE miRNAs were observed in the heat-tolerant variety GXN over the heatsensitive variety HJX during heat stress and these results may partially explain the difference in heat tolerance between the two genotypes. By integrating genome-wide analysis of DE miRNAs with mapping of QTLs for heat tolerance at the flowering stage in GXN, limited candidate miRNAs and their target genes associated with heat tolerance in GXN were identified. Our data provide new insight into the mechanism of rice in tolerance to heat stress and the limited candidates identified in this study offer an important source for further functional analysis and molecular breeding for heat tolerance in rice.

\section{MATERIALS AND METHODS}

\section{Plant Materials}

Two rice varieties that exhibit contrasting sensitivity to heat stress were used for this study: the heat-tolerant variety GXN (indica) and heat-sensitive variety HJX (indica). Twenty-three chromosome single-segment substitution lines (SSSLs) derived from HJX (recipient) and GXN (donor; Zhang et al., 2004) were used for QTL mapping. Each SSSL contains only one chromosomal segment that is different from HJX. The SSSLs used in the present study are listed in Table S1.

\section{Estimation of the Length of Substituted Chromosome Segment in a SSSL}

The lengths of substituted chromosome segments in single segment substitution lines were calculated based on their graphical genotypes (Young and Tanksley, 1989; Hospital, 2002). A chromosome segment flanked by two markers of donor type (DD) is considered as $100 \%$ donor type, a chromosome segment flanked by two markers of recipient type (RR) is considered as $0 \%$ donor type, and a chromosome segment flanked by one marker of donor type and one marker of recipient type (DR) is considered as $50 \%$ donor type. Therefore, the length of DD 
plus the length of two half DR is considered as the length of a substituted chromosome segment.

\section{Evaluation of Heat Tolerance}

Thirty plants per line were transplanted in cylindrical plastic pots $(11 \mathrm{~cm}$ in diameter $\times 23 \mathrm{~cm}$ in height). Single plant was planted in one pot. Each cylindrical plastic pot was filled with $1.00 \mathrm{~kg}$ of dry fine soil and $0.64 \mathrm{~g}$ of compound fertilizer $(\mathrm{N}$ $\left.\mathrm{P}_{2} \mathrm{O}_{5}-\mathrm{K}_{2} \mathrm{O}, \mathrm{N}: \mathrm{P}: \mathrm{K}=12: 18: 15\right)$. After the transplanted seedlings established and grew up to the tillering stage, each pot was applied with $0.33 \mathrm{~g}$ of compound fertilizer. Extra tillers were removed, leaving two tillers per plant to avoid overcrowding and to ensure better growth. At the heading stage, when the panicle exserted about $2 \mathrm{~cm}$ from the auricle, the plant was used for heat stress treatment. Twenty uniform plants per line were selected and divided into two groups with 10 plants each. The two groups of plants were transferred into two Conviron PGV36 growth chambers (Controlled Environments Ltd., Winnipeg, Canada), respectively. The photon flux density and relative humidity in the growth chambers were $200 \mu \mathrm{mol} \mathrm{m}{ }^{-2} \mathrm{~s}^{-1}$ (6:00-18:00) and $75 \pm 5 \%$, respectively. The temperature of the growth chamber for control was $28.0 / 22.0^{\circ} \mathrm{C}$ (day/night), while the average temperature of the growth chamber for heat stress was $31.0^{\circ} \mathrm{C}$. The concrete day and night temperature parameters for heat treatment were shown in Figure S1. After treated for 7 days in the growth chambers, the plants were taken back to the screen house for normal growth until maturity. At maturity, the following traits of tested plants were measured: total number of spikelets per plant (TSP) and the number of filled spikelets per plant (FSP). Spikelet fertility was expressed as SFP $=$ FSP/TSP $\times 100 \%$. The heat tolerance index $(\mathrm{HI})$ was used to evaluate heat tolerance, $\mathrm{HI}=$ heat treated SFP/control SFP. Three independent experiments were conducted.

\section{QTL Analysis}

The SSSLs derived from the heat-tolerant variety GXN (donor parent) and the heat-sensitive variety HJX (recurrent parent) were used to detect QTLs for heat tolerance. Theoretically, there is only a single segment difference between each SSSL and HJX. The detection of QTL for heat tolerance and its effect were based on the difference in heat tolerance between each SSSL and HJX. $T$-test was used to analyze the significance of difference using the program SPSS 12.0. A QTL was declared when the significance level was <0.05. According to Eshed and Zamir (1995), QTL additive effect $=($ phenotype of SSSL - phenotype of HJX)/2; QTL additive effect percentage $=$ additive effect/phenotype of $\mathrm{HJX} \times 100 \%$.

\section{Heat Stress Treatment and Sampling}

Mature seeds of GXN and HJX were incubated at $49^{\circ} \mathrm{C}$ for 4 days prior to germination, and then sown in pots with fine soil. The growth condition of the rice plants were the same as described in the evaluation of heat tolerance with some modifications. The control growth chamber was maintained at $27^{\circ} \mathrm{C}$ with a $12 \mathrm{~h}$ light/12 h dark photoperiod, and the growth chamber for heat treatment was maintained at $38^{\circ}$ Cwith a $12 \mathrm{~h}$ light $/ 12 \mathrm{~h}$ dark photoperiod. Panicle samples from both heat-treated and control plants were collected at 1, 6, and $24 \mathrm{~h}$ after heat treatment. Three biological replicates were conducted in the present study. All collected samples were immediately frozen in liquid nitrogen and stored at $-70^{\circ} \mathrm{C}$.

\section{Total RNA Isolation and Small RNA Deep Sequencing}

Samples of three biological replications were pooled together for small RNA sequencing. Total RNA from heat treated and control samples of GXN and HJX was isolated with Trizol (Invitrogen, Carlsbad, CA) according to the manufacturer's instruction. Small RNA library preparation and sequencing were performed with Solexa Sequencing Technology (ANNOROAD, Beijing, China). For construction of small RNA libraries, total RNA was fractionated by $15 \%$ polyacrylamide gel electrophoresis, and small RNAs in the range of 18-30 nt were purified. After dephosphorylation and ligation of a pair of Solexa adaptors to the $5^{\prime}$ and $3^{\prime}$ ends, sRNAs were then reverse-transcribed and amplified by PCR. The raw sequencing data has been submitted to NCBI (http://www.ncbi.nlm.nih.gov/) with accession number SRX834694.

\section{Characterization of Known miRNAs from the Sequencing Data}

All sequenced reads were mapped to the Oryza sativa genome (MSU7), using the Bowtie program (Li and Durbin, 2009) with version 1.0 .0 (-p 16, -f, -n 1, -e 80, - 18 , -a, -m 5, -best, -strata). Only reads less than five perfect matches to the genomic positions were reported. Reads were then searched against miRNA database (miRbase v20) for perfect matches using the miRDeep2 program (Friedländer et al., 2012) with version 2_0_0_5 (-t Rice) and defined miRNAs as "confirmed" when the mature sequence was observed 10 times or more in either library.

\section{Identification of Differentially Expressed miRNAs and Functional Analysis of Their Targets}

All reads were normalized as transcripts per million (TPM) according to the total sequenced reads in each sample. miRNAs with $\log _{2}$ Fold change $\geq 1.0$ or $\log _{2}$ Fold change $\leq-1.0, P \leq 0.05$, and FDR $\leq 0.05$ were considered to be significantly differentially expressed. Fold change $=\mid \mathrm{TPM}$ ratio $\mid$ (treatment/control). Fisher's exact test was used to calculate the $P$-value and false discovery rate (FDR) was estimated using Benjamini and Hochberg method in R package. The psRobot_tar with parameters (-ts 2, -gl 10) in program psRobot (version 1.2; Wu et al., 2012) version 1.2 was used for target gene prediction and an online server of psRNATarget (Dai and Zhao, 2011) (http://plantgrn.noble.org/psRNATarget/) was also used for target gene prediction with expectation range 2.0-3.0 and other default parameters when no results were predicted by using psRobot. PlantGSEA (Yi et al., 2013) was used for enrichment analysis of biological processes in Gene Ontology and Plant Ontology. KEGG pathway analysis was carried out on KEGG website (http://www.genome.jp/kegg/). 


\section{Quantitative Real-Time Polymerase Chain Reaction}

Real-time quantification of microRNAs by stem-loop RT-PCR was performed as described by Chen et al. (2005) and VarkonyiGasic et al. (2007). RNA RT reactions were performed using the primescript $^{\mathrm{TM}}$ RT reagent kit (Takara, Japan). Real-time PCR was carried out using the SYBR Premix Ex Taq ${ }^{\mathrm{TM}}$ kit (Takara, Japan), following the manufacturer's instructions, on a Biorad CFX Connect Real-Time System. The 5srRNA and EF1 $\alpha$ were used as endogenous normalized genes for miRNA and mRNA, respectively. All reactions were run in triplicate. Primers used to amplify the selected genes are listed in Table S2.

\section{5' RLM-RACE}

Total RNA was extracted from rice panicles. Poly(A) ${ }^{+}$mRNA was subsequently enriched using the Oligotex mRNA mini Kit (Qiagen, Germany). 5' rapid amplification of cDNA ends (5' RACE) was carried out with GeneRacer Kit (Invitrogen, America) as described by Sunkar et al. (2005). The first round PCR was performed with GeneRacer $5^{\prime}$ primer and gene-specific primer, followed by second round PCR with Gene-Race $5^{\prime}$ nested primer and gene-specific nested primer. The PCR products were gel-purified, cloned, and sequenced. The primers used in this study were shown in Table S2.

\section{Development of miR169r-5p Transgenic Plants and Evaluation of Heat Tolerance of Transgenic Plants}

For the Ubi:miR169r-5p construct, the precursor sequence of miR169r-5p was amplified from heat-tolerant rice variety GXN by PCR using the following primers: forward primer, 5'- GGTCAACCCAAATAAGCAAG -3'; reverse primer, $5^{\prime}$ - GAATAATACAGTGTAGCCATAGAGG - $3^{\prime}$. The DNA product was inserted into $\mathrm{POX}$ which harbors an ubiquitin promoter, and the resultant vector was electroporated into Agrobacterium tumefaciens EHA105. Rice transformation was performed as described by Toki et al. (2006). Evaluation of heat tolerance of the transgenic plants was the same as indicated above, the transgenic plants were treated by heat stress for 4 days and then taken back to the screen house for normal growth.

\section{RESULTS}

\section{Performance of the Two Selected Rice Genotypes under Heat Stress}

To screen for rice varieties with contrasting heat tolerance at the flowering stage for this study, we have evaluated the heat tolerance of rice germplasm from 11 countries at the flowering stage. Our results showed that GXN, an indica variety from China exhibited strong heat tolerance, whereas HJX, an indica variety from China was sensitive to heat stress (Figure S2). After heat treatment at the flowering stage, the spikelet fertility of GXN and HJX was 55.8 and $15.3 \%$, respectively (Table 1). The heat tolerance index $(\mathrm{HI})$ of $\mathrm{GXN}$ is 0.84 , much higher than that of HJX (0.20) and similar to N22, a well-known heat-tolerant indica landrace variety (Jagadish et al., 2010), with a HI of 0.85 in the present study (Table 1). Thus, GXN is a strong heat-tolerant variety while $\mathrm{HJX}$ is a heat-sensitive variety and their contrasting phenotypes in heat tolerance make them to be ideal materials for exploring the functional genes and molecular mechanisms of heat tolerance in rice.

\section{Genome-Wide Analysis of Small RNAs in Heat Treated and Untreated GXN and HJX at the Flowering Stage}

To identify the miRNAs at flowering stage in heat treated and untreated GXN and HJX, rice plants of the two genotypes at the flowering stage were subjected to heat stress treatment for 1 , 6 , and $24 \mathrm{~h}$, respectively. Small RNA libraries were constructed from panicle tissues in the heat-treated and untreated rice plants at the given time points. In total, 12 libraries were constructed and subjected to high-throughput sequencing analysis. After trimming the adaptor sequences, total sequencing reads of the 12 libraries ranged from 22.6 to 29.6 million, with the distinct (unique) reads that perfectly matched the rice genome ranged from 4.6 to 8.1 million (Table S3). Reads ranged in size from 18 to $30 \mathrm{nt}$, with peaks at 21 and $24 \mathrm{nt}$ (Figure S3), consisting with the previous analysis of small RNAs at the seedling stage in rice (Jeong et al., 2011).

Overall, we detected the expression of 446 known miRNAs representing 182 families (Table S4). Among them, 412 miRNAs belonging to 152 families (Table S4) were expressed both in GXN and HJX. Nineteen miRNAs belonging to 17 miRNA families were expressed specifically in GXN, whereas 15 miRNAs belonging to 13 miRNA families were expressed specifically in HJX (Table S5). Both in GXN and HJX, the most abundant expressed miRNA was miR396f-5p, with the reads reached to 958,958 in the library of GXN-1hT (Table S4). Interestingly, 42 miRNAs showed significantly higher basal level $(P \leq 0.05$ and $\log _{2}$ fold change $\geq 1.0$ ) in GXN than that in HJX in at least one of the three comparisons before heat stress (Table S6). The 42 miRNAs include those miRNAs belonging to the highly conserved miRNA families such as miR160, miR166, miR167, and miR168.

\section{The Expression Patterns of the Heat Responsive miRNAs in GXN and HJX}

Overall, 102 miRNAs were differentially expressed (DE) $\left(\log _{2}\right.$ fold change $\geq 1.0$ or $\log _{2}$ fold change $\leq-1.0, P \leq 0.05$, and FDR $\leq 0.05$ ) afterheat stress treatment in GXN and HJX (Figure 1A, Table S7). They could be classified into four categories: (1) codown-regulated miRNAs in both GXN and HJX; (2) co-regulated miRNAs but showed opposite expression patterns between GXN and HJX; (3) DE miRNAs specifically identified in GXN; (4) DE miRNAs specifically identified in HJX (Table S7). A total of 85 miRNAs were differentially expressed in GXN in at least one comparison, and 26 DE miRNAs were identified in HJX (Table S7). Fifteen miRNAs were differentially expressed at $1 \mathrm{~h}$ after heat stress in GXN, whereas no miRNA was differentially expressed at $1 \mathrm{~h}$ after heat stress in HJX (Figure 1A). These observations indicated that there were more DE miRNAs and the response 
TABLE 1 | The heat tolerance indexes of GXN, HJX, and N22.

\begin{tabular}{lccr}
\hline Variety & $\begin{array}{c}\text { Spikelet fertility (\%) } \\
\text { control }\end{array}$ & $\begin{array}{c}\text { Spikelet fertility(\%) } \\
\text { heat stress }\end{array}$ & $\begin{array}{c}\text { Heat tolerance } \\
\text { index }\end{array}$ \\
\hline HJX & $76.6 \pm 3.84$ & $15.3 \pm 0.91$ & $0.2 \pm 0.01$ \\
GXN & $66.4 \pm 4.97$ & $55.8 \pm 6.11$ & $0.84 \pm 0.09$ \\
N22 & $68.7 \pm 6.37$ & $58.67 \pm 3.86$ & $0.85 \pm 0.06$ \\
\hline
\end{tabular}

${ }^{a}$ The value is the mean of 10 plants.

of miRNAs to heat stress was earlier in the heat-tolerant variety GXN compared to the heat-sensitive variety HJX.

Among the 102 DE miRNAs, 9 miRNAs were observed both in GXN and HJX (Figure 1B, Table S7). Four of these miRNAs (miR164d, miR166i-3p, miR168a-3p, and miR397b) exhibited the same suppressed expression pattern after heat stress in both of the two rice varieties (Figure 1C), suggesting a persistent regulatory role of these miRNAs in heat tolerance. Furthermore, it is noteworthy that among the 102 DE miRNAs, only 14 of them were up-regulated after heat stress, including the miRNAs which were firstly up-regulated but then down-regulated during heat stress (Table S7). The other DE miRNAs were uniformly downregulated after subjected to heat stress, indicating that most DE miRNAs were under-presented under heat stress at the flowering stage in rice.

\section{Target Prediction and Functional Classification of Heat Responsive miRNAs}

DE miRNAs have the potential to regulate target transcripts to exert their functions in rice. To understand the functions and their potential regulatory roles of DE miRNAs in heat tolerance, we searched for the putative targets of the 102 DE miRNAs and performed Gene Ontology (GO) enrichment analysis of these predicted targets. Totally, 446 target genes were identified for the $102 \mathrm{DE}$ miRNAs (Table S8). The GO enrichment analysis suggested that metabolism, biosynthesis, and biological regulation were the most significant biological processes (Figure 2A, Table S9). Moreover, these significantly related biological processes are common between the two rice varieties, further suggesting the important roles of these miRNAs in heat tolerance. However, as shown in Figure 2A, the number of the target genes involved in these biological processes in GXN is much more than that in HJX. On the other hand, some biological processes such as RNA biosynthesis and DNAdependent transcription are GXN-specific (Figure 2A).

To further understand their functions of the DE miRNAs identified in this study, PO (Plant Ontology) enrichment analysis of the putative targets of the 102 DE miRNAs was performed. The results showed that among the nine tissues tested, the number of target genes in six tissues in GXN was significantly more than that in HJX (Figure 2B, Table S10). Particularly, "inflorescence" and "shoot system" had the largest number of target genes (Figure 2B). In addition, the target genes in "flower" and "gynoecium" were GXN specific (Figure 2B). Among the miRNAs involved, the previously identified heat responsive miRNA miR166 (Xin et al., 2010) was included. In addition to $H D-Z I P$ transcription factors, we identified that miR166a-3p is also predicted to target other two START domain containing proteins which are speculated to be involved in gynoecium development (Figure 3). RLM-RACE validated that these genes could be successfully cleaved by miR166a-3p. These results imply that miR166-mediated cleavage of the two target mRNAs might be involved in gynoecium development and heat tolerance at the flowering stage in rice.

\section{Validation of RNA Sequencing Data in this Study Using Quantitative RT-PCR}

To confirm the authenticity of the sequencing results, eight DE miRNAs were selected for expression analysis using quantitative RT-PCR (qRT-PCR). The eight miRNAs (miR159a.1, miR164a, miR160a-5p, miR166f, miR168a-5p, miR397b, miR398b, and miR528-5p) all have been reported to be involved in responses to heat stress or other abiotic stresses in plants (Xin et al., 2010; Zhou et al., 2010; Jeong et al., 2011; Chen et al., 2012; Guan et al., 2013; Fang et al., 2014; Stief et al., 2014). The quantitative RT-PCR assays revealed that the selected DE miRNAs exhibited different or the same expression patterns in GXN and HJX (Figure 4). The results showed an excellent concordance with the sequencing data (Figure 5; $R^{2}=0.8362$ ). As expected, the transcription of the target genes was negatively correlated with the abundance of their corresponding miRNAs (Figure 4). Thus, these results together indicate the reliability of the RNA sequencing data in the present study.

\section{Integrating Differential Expression Analysis of miRNAs with QTL Mapping to Identify miRNAs and Their Target Genes Associated with Heat Tolerance in Rice}

To cut down the number of candidate miRNAs and their target genes associated with heat tolerance, we performed QTL mapping and integrating QTL mapping with analysis of differential expression of miRNAs. Here, we used chromosome single-segment substitution lines (SSSLs) for identification of QTLs associated with heat tolerance. The SSSLs were developed from GXN (donor) and HJX (recurrent parent; Zhang et al., 2004). Through evaluation of the heat tolerance of the SSSLs and their recurrent parent at the flowering stage, we identified four QTLs for heat tolerance on chromosomes 3, 6, 8, and 12 across three independent experiments, designated as qHT-3, qHT-6, $q H T-8, q H T-12$, and they accounted for 19.32, 30.71, 51.67, and $14.47 \%$ of phenotypic variation, respectively (Table 2). These effective alleles are all from the heat-tolerant variety GXN. We looked for those DE miRNAs whose target genes are located in the QTL regions. We found that 8 target genes of the $26 \mathrm{DE}$ miRNAs belonging to 6 miRNA families were identified in the four QTL regions (Table 3).

To find the most possible target genes involved in heat tolerance in GXN among these 8 genes within the QTL regions, the transcription levels of the 8 genes in GXN and HJX both before and after heat stress were analyzed using qRT-PCR. The results (Figure 6A) revealed that LOC_Os08g31390 and LOC_Os12g43900 were not expressed. LOC_Os06g07530 and LOC_Os08g33630 exhibited similar expression patterns 


\section{A}

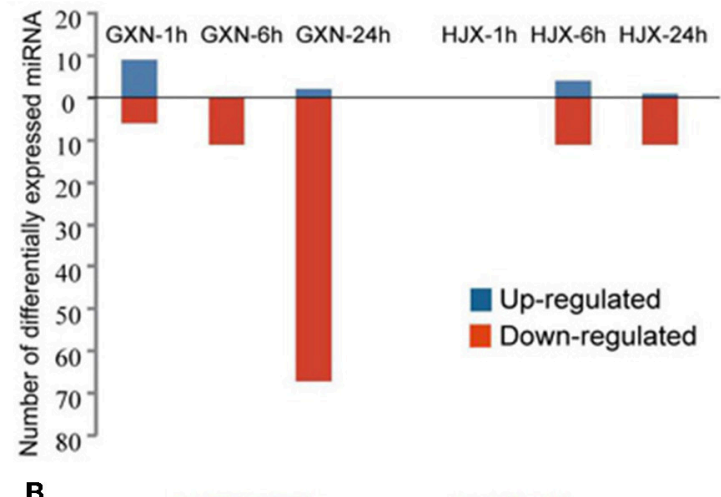

B

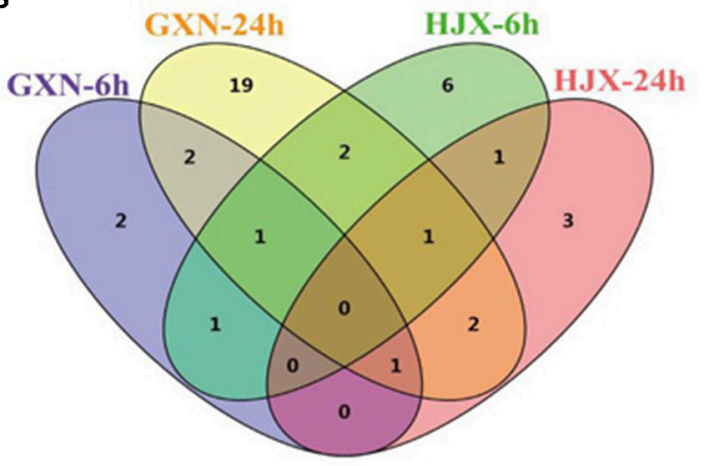

C

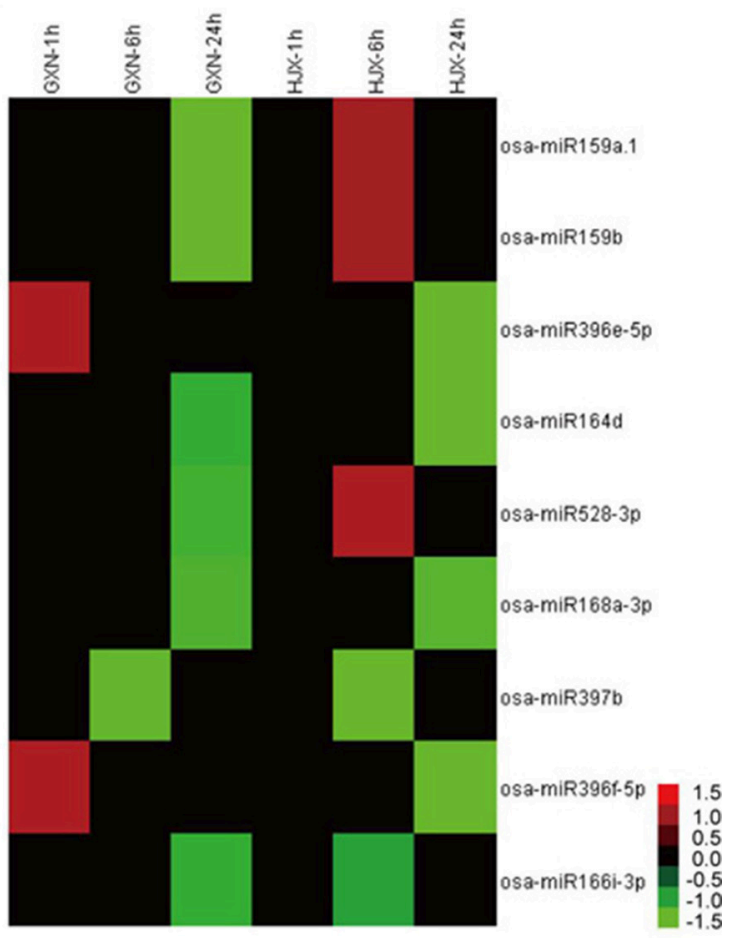

FIGURE 1 | The expression patterns of 102 differentially expressed (DE) miRNAs $\left(\log _{2}\right.$ fold change $\geq 1.0$ or $\log _{2}$ fold change $\leq-1.0 ; P \leq 0.05$ and FDR $\leq \mathbf{0 . 0 5}$ ) under heat stress. (A) The number of miRNAs that were up-regulated or down-regulated in GXN and HJX under heat stress. (B) Number of DE miRNA families in four sequencing libraries. Venn diagram was created using VENNY. (C) Expression patterns of 9 DE miRNAs under heat stress both in GXN and HJX.

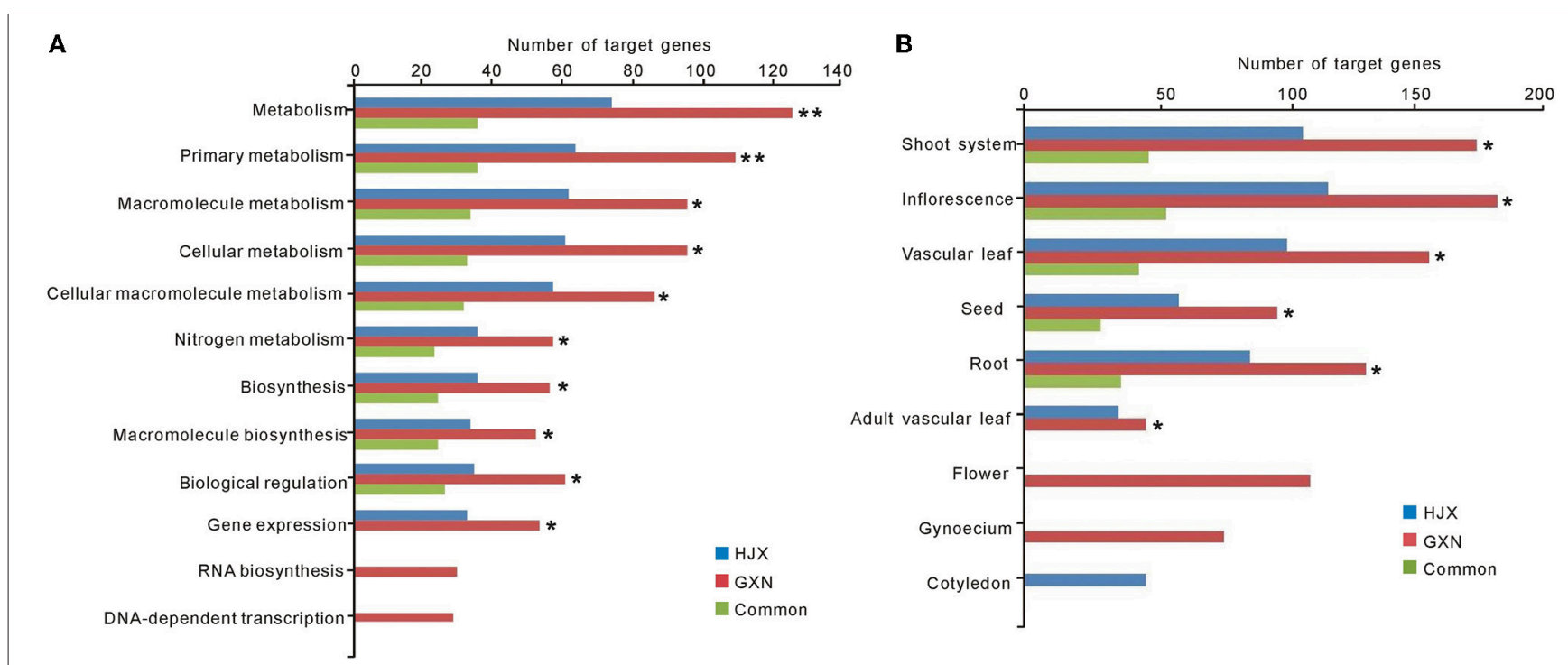

FIGURE 2 | GO and PO slims of functional categorization of the predicted targets of 102 DE miRNAs. ${ }^{*}$ and * indicate significant differences in the number of target genes between GXN and $\mathrm{HJX}$ at $P \leq 0.01$ and $P \leq 0.05$, respectively. (A) GO enrichment analysis of the predicted targets of $102 \mathrm{DE}$ miRNAs. (B) PO enrichment analysis of the predicted targets of $102 \mathrm{DE}$ miRNAs.

both in GXN and HJX. However, LOC_Os08g34740 (SGT1) and LOC_Os08g34900, (pectinesterase) within qHT-8, LOC_Os12g42190 and LOC_Os12g42400 within qHT-12 all showed differential expression under heat stress between GXN and HJX, suggesting their probable roles in heat tolerance. MiR169 and its target gene LOC_Os12g42400 (Os12g0618600 


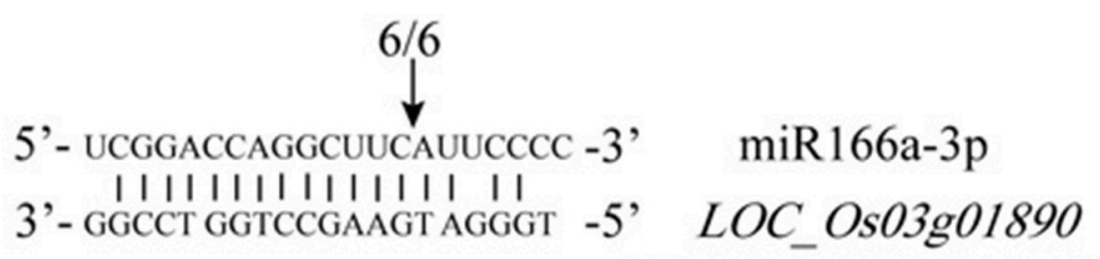

5'- UCGGACCAGGCUUCAUUCCCC -3,
3IIIIIIIIIIII II
3'-GGCCT GGTCCGAAGT AGGGT

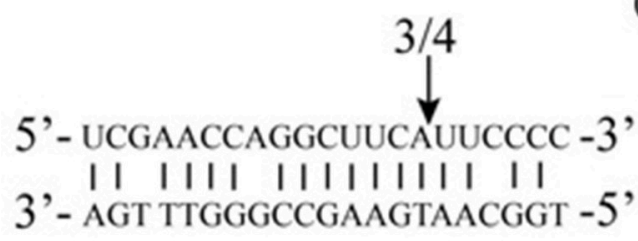

(START domain containing protein)
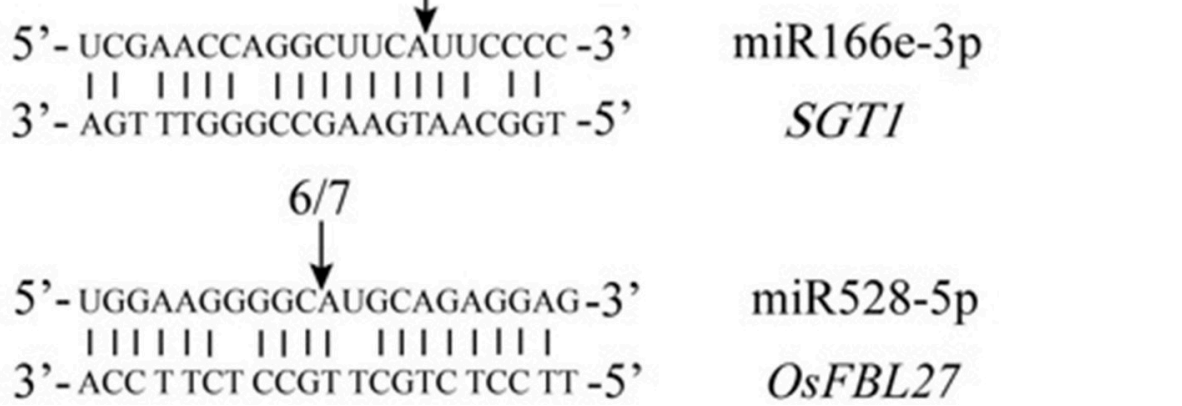

FIGURE 3 | Validation of predicted target genes by modified RLM 5' -RACE. The arrows indicate the cleavage sites. The number of cloned RACE products that were sequenced is shown above each sequence.

in RAP locus) have been reported to be ABA-responsive (Tian et al., 2015) and it has been well-known that ABA plays a pivotal role in adaptive responses to various abiotic and biotic stresses (Lee and Luan, 2012). SGT1 (the target of miR166e-3p; Figure 4) can bind to heat shock proteins (HSPs) such as HSP90 and HSP70 and has been demonstrated as a positive regulator in plant and mammalian cells for the response to thermal stress (Arya et al., 2007; Mayor et al., 2007; Zabka et al., 2008; Shirasu, 2009; Prus and Filipek, 2011). Transcription analysis revealed that miR169r-5p and miR166e-3p exhibited negative correlation expression patterns with their target genes (SGT1, LOC_Os12g42400) under heat stress (Figure 6B). Furthermore, the signaling pathway analysis of the target genes of the DE miRNAs through KEGG (http://www.genome.ad.jp/kegg/) indicated that the gene pectinesterase (target of miR3980a/b-3p) within qHT-8 was involved in starch and sucrose metabolism which is related to pollen fertility and grain filling under heat stress (Wang et al., 2014).

\section{miR169r-5p Overexpressing Plants Showed Enhanced Heat Tolerance at the Flowering Stage in Rice}

Based on its possible role in heat tolerance and novelty, we selected miR169r-5p for further functional confirmation. The transgenic rice plants constitutively overexpressing miR169r-5p were produced in Zhonghual1 (ZH11), which is sensitive to heat stress at the flowering stage. The miR169r-5p overexpressing (OE) plants showed no morphological changes and were fertile. Three independent transgenic lines (OE1, OE2, and OE3) with higher miR169r-5p expression were identified by miRNA qRT-PCR compared to the wild-type ZH11 plants (Figure 7B). Consistently, the expression of its target gene LOC_Os12g42400 was reduced to $<35 \%$ of that in the control plants (Figure 7C). The spikelet fertility of the transgenic plants ranged from 53.23 to $58.06 \%$ (average of $55.79 \%$ ), significantly higher than that of ZH11 plants (42.50\%) (Figures 7A,D). Similarly, the heat tolerance indexes of transgenic plants were also significantly higher than that of $\mathrm{ZH} 11$ plants (Figure 7E).

To gain further insight into the mechanisms of the enhanced heat-tolerance of miR169r-5p overexpressing plants, the transcripts of four HSPs that have been reported to be involved in response to heat stress (Qin et al., 2015) were analyzed in the transgenic lines and control plants both before and after heat stress. The results showed that heat stress treatment significantly induced the transcription of these four HSPs both in the control and transgenic plants. However, the transcription levels of the four HSPs were all higher in miR169r$5 \mathrm{p}$ overexpressing plants than in control plants (Figure S4), further supporting the positive role of miR169r-5p in heat tolerance. 


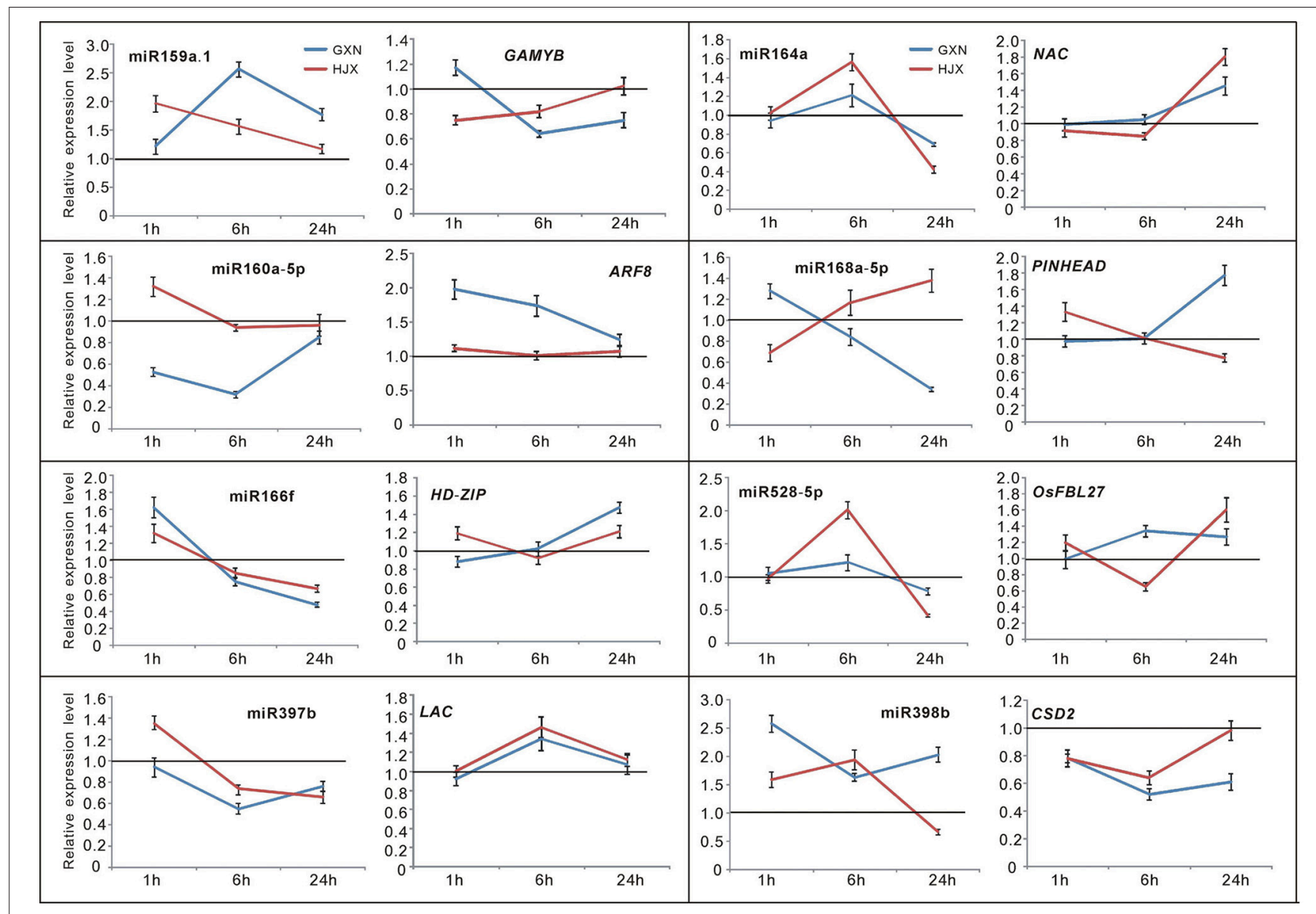

FIGURE 4 | Time-course expression profiling of the selected miRNAs and their target genes at 1, 6, and $24 \mathrm{~h}$ after heat stress treatment at the flowering stage in both heat-tolerant variety GXN and heat-sensitive variety HJX using RT-PCR. The expression of control plants without heat stress was set to "1.0" at different time points, and was marked by the black line. Bars represent means (three replicates) \pm standard derivation (SD).

\section{DISCUSSION}

\section{miRNAs Are Involved in Heat Tolerance at the Flowering Stage in Rice}

In the past few years, miRNAs have been validated to play crucial roles in biotic and abiotic stresses at the post-transcriptional level by targeting mRNAs for cleavage or repressing translation in many plants (Sunkar and Zhu, 2004; Sunkar et al., 2006; Song et al., 2013; Zhou et al., 2013). Recent studies have also uncovered the complexity of miRNAs in regulation of plants in responses to heat stress using high-throughput sequencing technology and demonstrated that miRNAs may function as important modulators in heat acclimation (Xin et al., 2010; Jeong et al., 2011; Yu et al., 2011; Chen et al., 2012). As one of the most important crops, rice plant is significantly affected by high temperature stress. The previous study has demonstrated that miRNAs play important role in heat tolerance at the seedling stage in rice (Jeong et al., 2011). However, genome-wide analysis of rice heat stress-regulated miRNAs has not been conducted at the flowering stage, which is very important for rice yield and is the most sensitive stage to heat stress (Matsui and Omasa,
2002; Prasad et al., 2006). In the present study, two parallel small RNA profiling experiments were performed at the flowering stage using Illumina sequencing technology in both heat-tolerant variety GXN and heat-sensitive variety $\mathrm{HJX}$. The small RNA expression was monitored at 1, 6, and $24 \mathrm{~h}$. Overall, 102 miRNAs were identified with at least two-fold change during heat stress in the present study. Compared with the previous studies, 63 of the 102 DE miRNAs except for miR3980 have been reported to play key roles during flower development in plants (Wu et al., 2006; Lee et al., 2010; Nag and Jack, 2010; Kim et al., 2011; Xia et al., 2012; Zhang et al., 2013; Spanudakis and Jackson, 2014). The suggested target gene of miR3980 is pectinesterase, which may be a key regulator in pollen fertility and grain filling. In the present study, we have confirmed the negative correlation between miR3980 and pectinesterase in expression (Figure 6A). These results suggest that heat stress can affect the expression of many miRNAs which are involved in flower development or fertility in rice. Based on these results, the module of the putative network of the seven heat-responsive miRNAs and their respective target genes involved in heat tolerance and flower development or fertility is presented in Figure 8. As flowering 


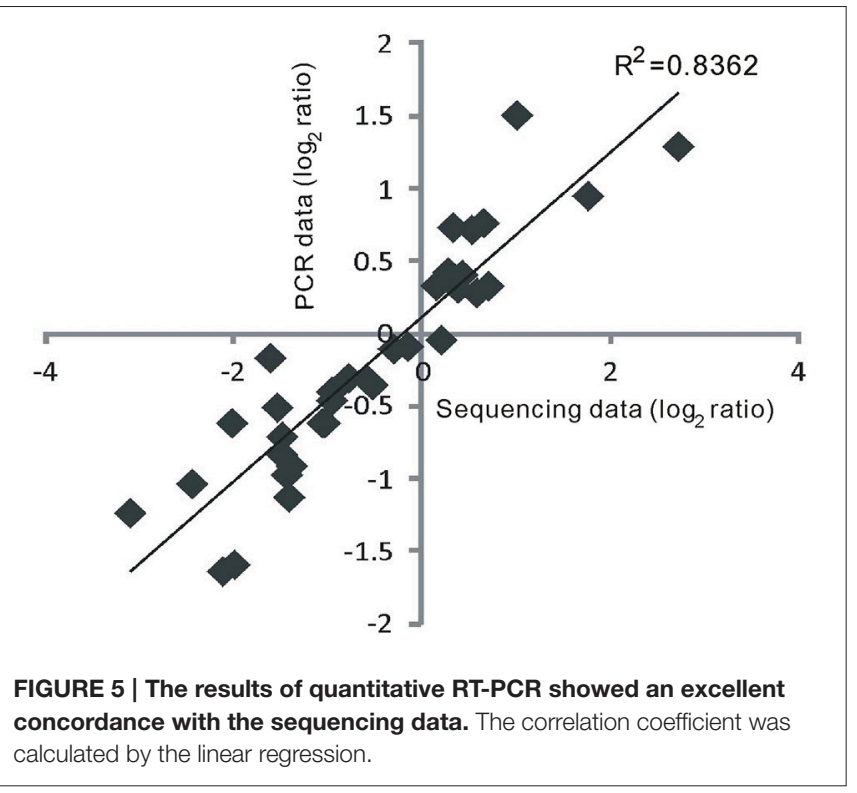

TABLE 2 | Heat-tolerant QTLs identified at the flowering stage.

\begin{tabular}{|c|c|c|c|c|c|}
\hline QTL & Chr & Marker intervals & $\begin{array}{l}\text { Length } \\
(\mathrm{Mb})\end{array}$ & $\begin{array}{l}\text { Additive } \\
\text { effect }^{*}\end{array}$ & $\begin{array}{l}\text { Additive effect } \\
\text { percentage }{ }^{\star}(\%)\end{array}$ \\
\hline qHT-3 & 3 & $\begin{array}{l}\text { RM227-RM565- } \\
\text { RM148-End }\end{array}$ & 1.03 & 3.59 & 19.32 \\
\hline$q H T-6$ & 6 & $\begin{array}{l}\text { RM190-RM587- } \\
\text { RM510-RM225- } \\
\text { RM217-RM50 }\end{array}$ & 1.80 & 5.60 & 30.71 \\
\hline$q H T-8$ & 8 & $\begin{array}{l}\text { RM404-RM339- } \\
\text { RM515-RM223- } \\
\text { RM531-RM210-RM149 }\end{array}$ & 6.86 & 9.24 & 51.67 \\
\hline qHT-12 & 12 & $\begin{array}{l}\text { RM235-RM17- } \\
\text { PSM193-End }\end{array}$ & 0.96 & 2.72 & 14.47 \\
\hline
\end{tabular}

*The value is the mean of three independent experiments.

stage is a critical stage for plant fitness and crop yield (Huijser and Schmid, 2011), a detailed understanding of the involvement of miRNAs in heat tolerance at the flowering stage is essential for yield improvement in agricultural practice.

Like miRNAs, small interfering RNAs (siRNAs) have also been demonstrated to play important roles in stress tolerance (Khraiwesh et al., 2012). In our sequencing data, the group with $24 \mathrm{nt}$ was the largest size group for the small RNAs. Since canonical heterochromatic siRNAs are $24 \mathrm{nt}$, this result implies that siRNAs may be also involved in rice heat tolerance. However, further study is needed to see if siRNAs participate in regulation of rice in response to heat stress.

\section{There Are Convergent and Divergent Mechanisms of miRNA in Regulation of Heat Stress Response in Plants}

In this study, we identified that 16 miRNAs of the 102 DE miRNAs were up-regulated whereas the others were all
TABLE 3 | Differentially expressed miRNAs with target genes located in the QTL regions.

\begin{tabular}{|c|c|c|c|}
\hline QTL & miRNA & ID of target genes & $\begin{array}{l}\text { Target gene } \\
\text { description }\end{array}$ \\
\hline$q H T-6$ & osa-miR528-3p & LOC_Os06g07530 & $\begin{array}{l}\text { Retrotransposon } \\
\text { protein, putative }\end{array}$ \\
\hline \multirow[t]{4}{*}{$q H T-8$} & osa-miR5794 & LOC_Os08g31390 & $\begin{array}{l}\text { Retrotransposon } \\
\text { protein }\end{array}$ \\
\hline & osa-miR166a/c/e-5p & LOC_Os08g33630 & $\begin{array}{l}\text { UPF0016 domain } \\
\text { containing protein }\end{array}$ \\
\hline & osa-miR166e-3p & LOC_Os08g34740 & SGT1 protein \\
\hline & osa-miR3980a/b-3p & LOC_Os08g34900 & Pectinesterase \\
\hline \multirow[t]{7}{*}{$q H T-12$} & osa-miR319a-3p.2-3p & LOC_Os12g42190 & Transposon protein \\
\hline & osa-miR319b & LOC_Os12g42190 & Transposon protein \\
\hline & osa-miR169a/h/j/k/l/m & LOC_Os12g42400 & $\begin{array}{l}\text { Nuclear transcription } \\
\text { factor } Y \text { subunit }\end{array}$ \\
\hline & osa-miR169i-5p.1 & LOC_Os12g42400 & $\begin{array}{l}\text { Nuclear transcription } \\
\text { factor } Y \text { subunit }\end{array}$ \\
\hline & osa-miR169r-5p & LOC_Os12g42400 & $\begin{array}{l}\text { Nuclear transcription } \\
\text { factor } Y \text { subunit }\end{array}$ \\
\hline & osa-miR166a/b/c/d/e/i/j-3p & LOC_Os12g43900 & $\begin{array}{l}\text { Retrotransposon } \\
\text { protein, putative }\end{array}$ \\
\hline & osa-miR166f & LOC_Os12g43900 & $\begin{array}{l}\text { Retrotransposon } \\
\text { protein, putative }\end{array}$ \\
\hline
\end{tabular}

down-regulated by heat stress. This result is consistent with the previous analysis in Brassica rapa and Populus tomentosa that most identified miRNAs were down-regulated under heat stress (Yu et al., 2011; Chen et al., 2012). Interestingly, among the 102 DE miRNAs, 17 miRNA families including miR156, miR397, and miR398 have been demonstrated to be involved in heat stress response at the seedling stage in rice and other plant species (Xin et al., 2010; Jeong et al., 2011; Yu et al., 2011; Chen et al., 2012; Guan et al., 2013), further implying their important roles in heat stress response. The GO analysis of the target genes in the present study also showed that the most abundant GO items were metabolism, biological regulation, and biosynthesis, in keeping with the previous studies in wheat and Arabidopsis (Qin et al., 2008; Guan et al., 2013). These results indicated that there are common mechanisms of miRNAs in regulation of heat tolerance between different growth stages and among different plant species. However, compared with the previous study at the seedling stage (Xin et al., 2010; Jeong et al., 2011; Yu et al., 2011), we also identified different set of heat responsive miRNAs in the present study. For example, many miRNAs, such as miR156, miR159, miR160, and miR166, were up-regulated by heat stress in wheat (Xin et al., 2010), whereas these miRNAs were down-regulated in our study. MiR397b was induced by heat stress at the seedling stage in rice (Jeong et al., 2011) while it was down-regulated at the flowering stage. Moreover, 40 miRNAs were firstly reported to be involved in heat stress response in this study. These results indicated that there are different mechanisms of miRNAs in regulation of heat tolerance between different growth stages and among different plant species. Taken together, we can conclude that there are convergent and divergent 
A

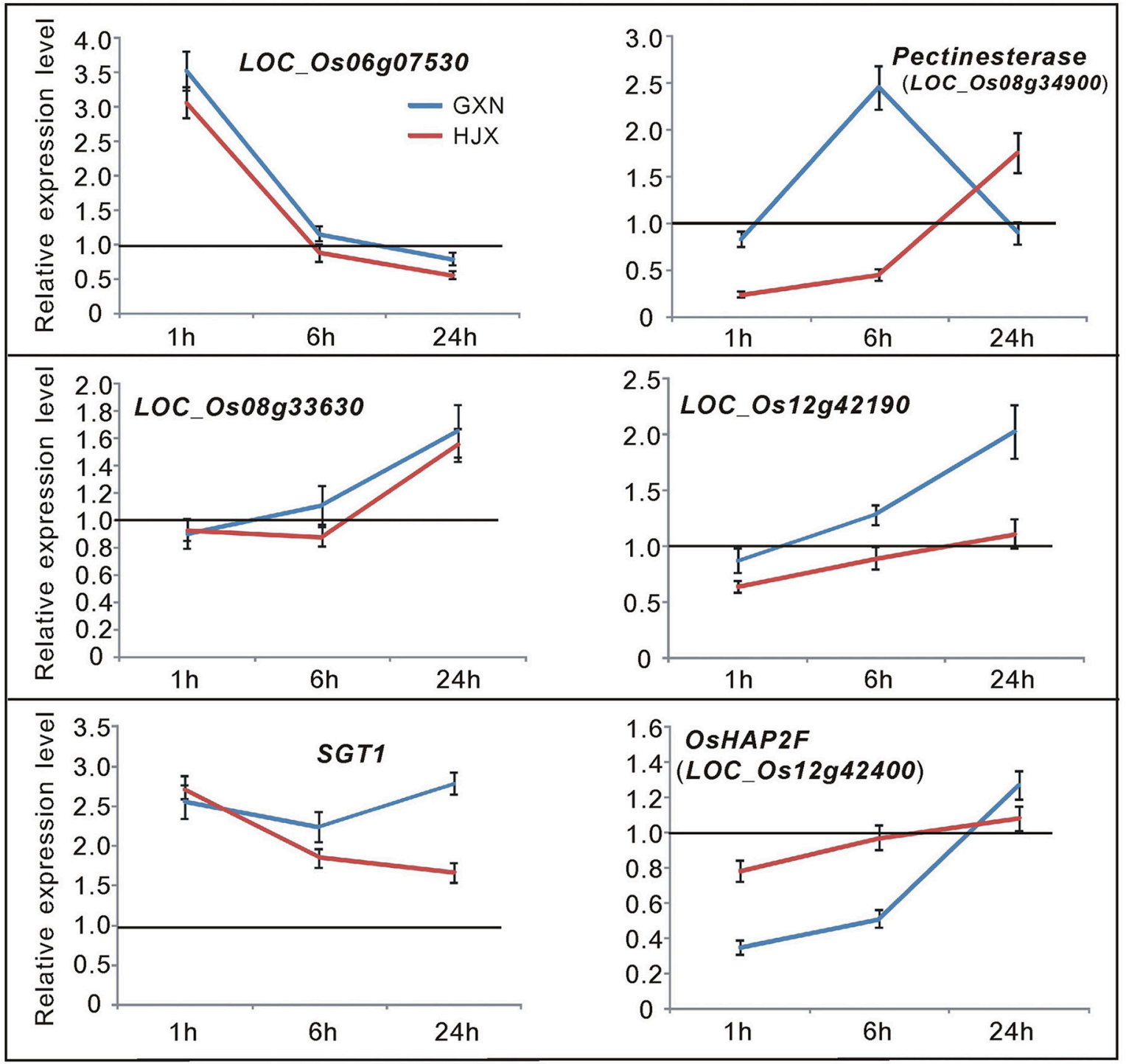

B

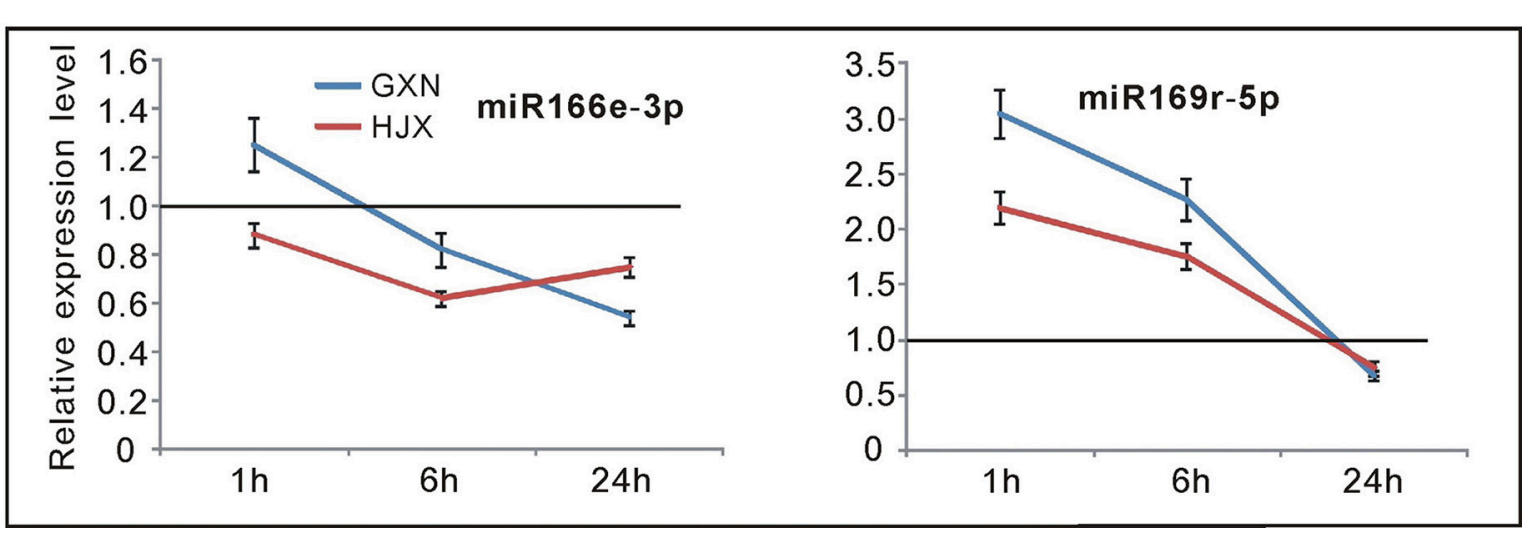

FIGURE 6 | Time-course expression profiling of the candidate miRNAs and their target genes (in the QTL regions) at 1, 6, and $24 \mathrm{~h}$ after heat stress treatment at the flowering stage in GXN and HJX. The expression of control plants without heat stress was set to "1.0" at different time points, and was marked by the black line. Bars represent means (three replicates) \pm SD. (A) Time-course expression profiling of 6 target genes in the QTL regions. (B) Time-course expression profiling of miR166e-3p and miR169r-5p. 


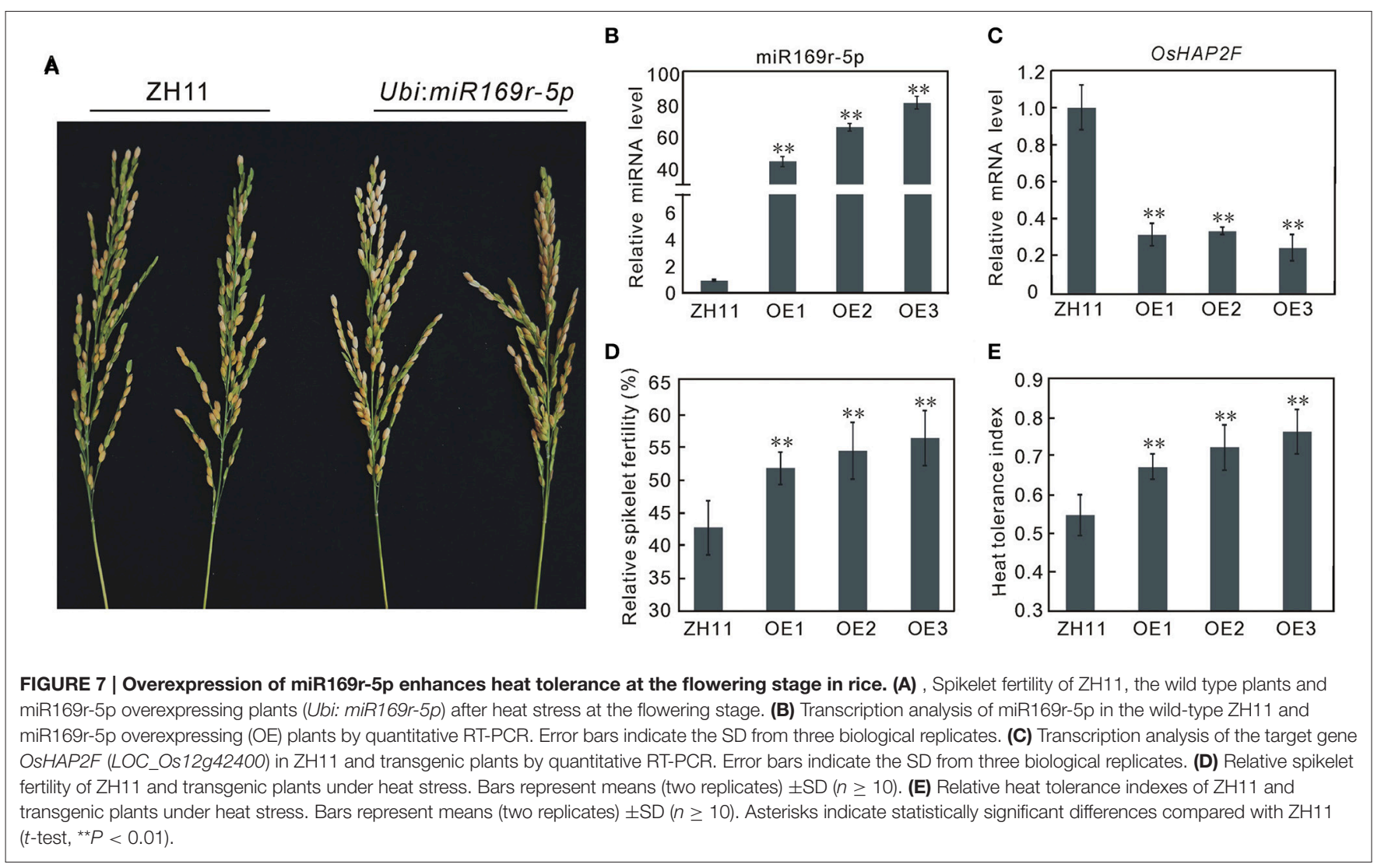

mechanisms of miRNA in regulation of heat stress response in plants.

\section{The Different Expression Patterns of miRNAs in GXN and HJX under Heat Stress May Contribute to Their Contrasting Heat Tolerance}

In previous studies, only one genotype is used to identify the heat stress responsive miRNAs in plants. To help better understand the mechanism of heat tolerance at flowering stage in rice, we used GXN and HJX, the two rice genotypes with contrasting heat tolerance for pairwise analysis of miRNAs. Our results revealed significant differences of miRNAs in expression between the two rice genotypes before and after heat stress. Firstly, a large number of conserved miRNAs (e.g., miR160, miR166, miR167, and miR168) exhibited higher expression level in the heat tolerant variety GXN over the heat sensitive variety HJX before heat stress. Secondly, more DE miRNAs were identified and the response of DE miRNAs to heat stress was much earlier in GXN than that in HJX. As shown in Table S7, 76 DE miRNAs were specifically expressed in GXN whereas only $17 \mathrm{DE}$ miRNAs were specifically identified in HJX. As miRNAs exert their functions through targeting mRNA transcripts, more DE miRNAs in heat-tolerant variety GXN resulted in an increased transcriptome variation than that in the heat-sensitive variety HJX. Specifically, the number of target genes involved in biological process or tissue development was much larger in GXN over HJX. Thirdly, the co-regulated miRNAs under heat stress also exhibited differential expression patterns between GXN and HJX. Among the nine co-regulated miRNAs, we identified that miR396e-5p and miR396f-5p were significantly up-regulated in the tolerant variety under heat stress but were down-regulated in the sensitive variety, suggesting they may positively regulate heat tolerance. In contrast, the expression of miR159a.1, miR159b, and miR528-3p was repressed in the tolerant variety under heat stress but were induced in the sensitive variety, implying they may negatively regulate heat tolerance. Furthermore, the four miRNAs (miR164d, miR166i-3p, miR168a-3p, and miR397b) all exhibited the same suppressed expression pattern in both the tolerant and sensitive varieties after heat stress treatment, implying that they may act in fundamental responses to heat stress. Except for heat stress, previous reports have indicated that the four miRNAs were also involved in other abiotic stresses, such as drought and salinity (Kansal et al., 2015; Xie et al., 2015), More importantly, miR164 also showed an similar response to drought stress in both tolerant and sensitive varieties (Kansal et al., 2015), further supporting its persistent regulatory role in abiotic stresses. Based on these results, we deduced that these differences might partially account for their contrasting heat tolerance of the two rice varieties. Similarly, the earlier studies have demonstrated that the heat-resistant tomato (Solanum lycopersicum L.) cultivar (cv. 3042) exhibited higher basal expression of HSP101 compared with the heat-susceptible 


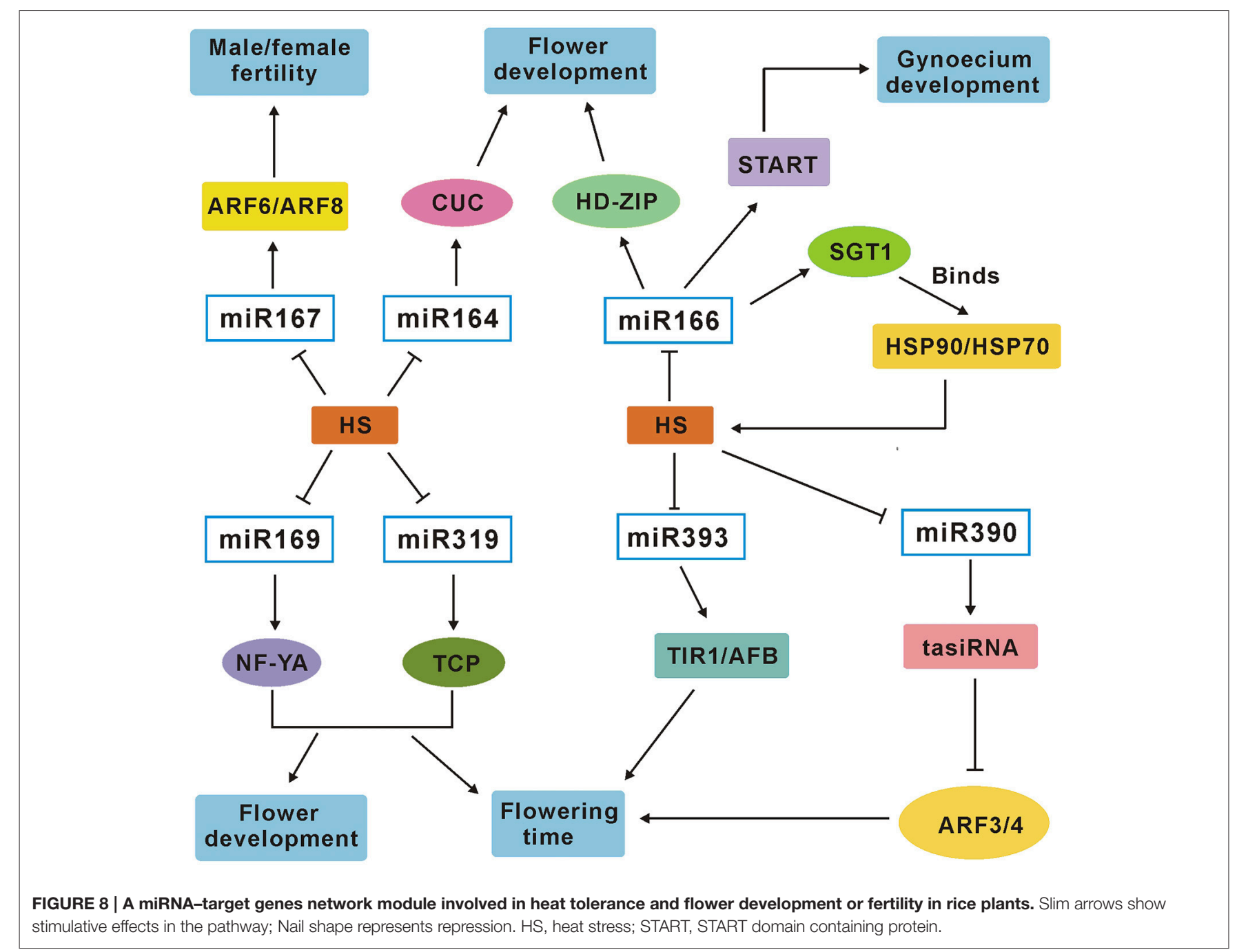

cultivar (cv. 3017) and constitutive expression of HSP101 could elevate the thermo-tolerance successfully (Katiyar-Agrawal et al., 2003; Pressman et al., 2007; Frank et al., 2009). Additionally, the previous study has indicated that the number of differentially expressed genes (DEGs) was higher in drought-tolerant rice than in drought-susceptible rice (Moumeni et al., 2011). Quicker and more efficient reversion of gene expression was also observed in chilling-tolerant genotype than in chilling-sensitive genotype (Zhang et al., 2012). These results together with our results suggest that higher basal expression of some important genes, more differentially expressed genes and quick response of the related genes may be the common features of tolerant genotypes over sensitive genotypes in response to abiotic stresses in rice.

\section{High-Throughput Sequencing Combined With QTL Mapping Provides an Efficient Way to Excavating Candidate miRNAs}

Though, RNA sequencing has been proved to be a powerful method in identifying miRNAs and their target genes associated with stress response (Xin et al., 2010; Jeong et al., 2011; Yu et al., 2011), it results in a large number of candidates. It is very difficult to pinpoint the miRNAs and genes that are important for the traits of interest. In the present study, we have identified 102 miRNAs that are significantly regulated by heat stress at the flowering stage. To screening for the important candidate miRNAs and genes that are involved in heat tolerance in rice, we employed RNA sequencing and QTL mapping. Two rice genotypes with contrasting heat tolerance were also used to help identify the candidate miRNAs and their target genes. By analyzing the significantly differentially expressed miRNAs between GXN and HJX under heat stress, together with analyzing the DE miRNAs whose targets are located in the QTL regions, we were able to successfully reduce the number of candidate miRNAs to 26 that belong to 6 miRNA families and identify 8 target genes within the QTL regions. These miRNA families include the three conserved miRNA families, miR166, miR169, and miR319, which have been confirmed to play pivotal roles in other stresses (Zhou et al., 2010; Ni et al., 2013; Li Y. et al., 2014). After differential expression analysis of the 8 target genes by RT-PCR, the number of candidate genes was further confined to four which showed differential 
expression between GXN and HJX under heat stress. Based on literatures and bioinformatics analysis, LOC_Os12g42400, SGT1 and pectinesterase are the most possible targets associated with heat tolerance at the flowering stage in rice. We identified that the transcription of miR169r-5p was significantly upregulated in both heat-tolerant variety GXN and heat-sensitive variety $\mathrm{HJX}$ at 1 and $6 \mathrm{~h}$ after heat stress treatment, but the expression levels in GXN were higher than that in HJX (Figure 6B). Accordingly, the transcription of its target gene LOC_Os12g42400 was significantly down-regulated by heat stress treatment in heat-tolerant variety GXN, but its expression is almost not changed when subjected to heat stress in heatsensitive variety HJX (Figure 6A). These results indicated the important roles of miR169r-5p and its target LOC_Os12g42400 in heat tolerance. Further, the miR169r-5p overexpressing plants, in which the transcription of LOC_Os12g42400 was significantly down-regulated, showed significantly higher spikelet fertility compared to wild type plants. Thus, the function of miR169r-5p on heat tolerance can be confirmed. Taken together, our results suggest that integrating RNA sequencing with QTL mapping is an efficient and cost-effective way to narrow down candidate miRNAs and target genes.

\section{AUTHOR CONTRIBUTIONS}

QL conducted the quantitative qRT-PCR assay, transgenic functional confirmation, drafting the manuscript and proposal

\section{REFERENCES}

Arya, R., Mallik, M., and Lakhotia, S. C. (2007). Heat shock genes-integrating cell survival and death. J. Biosci. 32, 595-610. doi: 10.1007/s12038-007-0059-3

Baker, J. T., Allen, L. H. Jr., and Boote, K. J. (1992). Temperature effects on rice at elevated CO2 concentration. J. Exp. Bot. 43, 959-964. doi: 10.1093/jxb/43.7.959

Boccara, M., Sarazin, A., Thiébeauld, O., Jay, F., Voinnet, O., Navarro, L., et al. (2014). Arabidopsis miR472-RDR6 silencing pathway modulates PAMP-and effector-triggered immunity through the post-transcriptional control of disease resistance genes. PLoS Pathog. 10:e1003883. doi: 10.1371/journal.ppat.1003883

Chen, C., Ridzon, D. A., Broomer, A. J., Zhou, Z., Lee, D. H., Nguyen, J. T., et al. (2005). Real-time quantification of microRNAs by stem-loop RT-PCR. Nucleic Acids Res. 33, e179. doi: 10.1093/nar/gnil78

Chen, L., Ren, Y., Zhang, Y., Xu, J., Sun, F., Zhang, Z., et al. (2012). Genome-wide identification and expression analysis of heat-responsive and novel microRNAs in Populus tomentosa. Gene 504, 160-165. doi: 10.1016/j.gene.2012.05.034

Dai, X., and Zhao, P. X. (2011). psRNATarget: a plant small rna target analysis server. Nucleic Acids Res. 39, W155-W159. doi: 10.1093/nar/gkr319

Eshed, Y., and Zamir, D. (1995). An introgression line population of Lycopersicon pennellii in the cultivated tomato enables the identification and fine mapping of yield- associated QTL. Genetics 141, 1147-1162.

Fang, Y., Xie, K., and Xiong, L. (2014). Conserved miR164-targeted NAC genes negatively regulate drought resistance in rice. J. Exp. Bot. 65, 2119-2135. doi: $10.1093 /$ jxb/eru072

Farrell, T. C., Fox, K. M., Williams, R. L., and Fukai, S. (2006). Genotypic variation for cold tolerance during reproductive development in rice: screening with cold air and cold water. Field Crops Res. 98, 178-194. doi: 10.1016/j.fcr.2006.01.003

Frank, G., Pressman, E., Ophir, R., Althan, L., Shaked, R., Freedman, M., et al. (2009). Transcriptional profiling of maturing tomato (Solanum lycopersicum L.) microspores reveals the involvement of heat shock proteins, ROS scavengers, hormones, and sugars in the heat stress response. J. Exp. Bot. 60, 3891-3908. doi: $10.1093 /$ jxb/erp234 writing. TFY conducted the QTL analysis for heat tolerance and TY conducted the bioinformatics analysis. SZ, XW, JZ, XM, and JD participated in RNA extraction and quantitative qRT-PCR assays. BL conceived of the study, drafted proposal, and corrected manuscript.

\section{FUNDING}

This research was supported by the National Youth Science Foundation of China (31201198), the Key Project of Guangdong Scientific and Technological Plan (2015B020231002), and the Guangdong Modern Agricultural Creation Team Project (2016LM2148).

\section{ACKNOWLEDGMENTS}

We thank Professor Guiquan Zhang in South China Agriculture University, China for providing the experimental materials. We also thank Dr. Congying Wang in Plant Protection Research Institute, Guangdong Academy of Agricultural Sciences, China for his valuable suggestions on manuscript preparation.

\section{SUPPLEMENTARY MATERIAL}

The Supplementary Material for this article can be found online at: http://journal.frontiersin.org/article/10.3389/fpls.2017. 00043/full\#supplementary-material

Friedländer, M. R., Mackowiak, S. D., Li, N., Chen, W., and Rajewsky, N. (2012) miRDeep2 accurately identifies known and hundreds of novel microRNA genes in seven animal clades. Nucleic Acids Res. 40, 37-52. doi: 10.1093/nar/ gkr688

Fujii, H., Chiou, T. J., Lin, S. I., Aung, K., and Zhu, J. K. (2005). A miRNA involved in phosphate-starvation response in Arabidopsis. Curr. Biol. 15, 2038-2043. doi: 10.1016/j.cub.2005.10.016

Guan, Q., Lu, X., Zeng, H., Zhang, Y., and Zhu, J. (2013). Heat stress induction of miR398 triggers a regulatory loop that is critical for thermotolerance in Arabidopsis. Plant J. 74, 840-851. doi: 10.1111/tpj.12169

He, H., Liang, G., Li, Y., Wang, F., and Yu, D. (2014). Two young MicroRNAs originating from target duplication mediate nitrogen starvation adaptation via regulation of glucosinolate synthesis in Arabidopsis thaliana. Plant Physiol. 164, 853-865. doi: 10.1104/pp.113.228635

Hospital, F. (2002). "Marker-assisted backcross breeding: a case-study in genotype building theory," in Quantitative Genetics, Genomics and Plant Breeding, ed M. S. Kang (Wallingford, UK: CABI Publishing), 135-140.

Huijser, P., and Schmid, P. (2011). The control of developmental phase transitions in plants. Development 138, 4117-4129. doi: 10.1242/dev.063511

IPCC (2007). "Summary for policymakers," in Climate Change 2007: Impacts, Adaptation and Vulnerability. Contribution of Working Group II to the Fourth Assessment Report of the Intergovernmental Panel on Climate Change, eds M. L. Parry, O. F. Canziani, J. P. Palutikof, P. J. van der Linden, and C. E. Hanson (Cambridge, UK: Cambridge University Press), 7-22.

Jagadeeswaran, G., Saini, A., and Sunkar, R. (2009). Biotic and abiotic stress down-regulate miR398 expression in Arabidopsis. Planta 229, 1009-1014. doi: 10.1007/s00425-009-0889-3

Jagadish, S. V., Craufurd, P. Q., and Wheeler, T. R. (2007). High temperature stress and spikelet fertility in rice (Oryza sativa L.). J. Exp. Bot. 58, 1627-1635. doi: $10.1093 /$ jxb/erm003

Jagadish, S. V., Muthurajan, R., Oane, R., Wheeler, T. R., Heuer, S., Bennett, J., et al. (2010). Physiologica and proteomic approaches to address heat 
tolerance during anthesis in rice (Oryza sativa L.). J. Exp. Bot. 61, 143-156. doi: $10.1093 / \mathrm{jxb} / \mathrm{erp} 289$

Jeong, D. H., Park, S., Zhai, J., Gurazada, S. G., De Paoli, E., Meyers, B. C., et al. (2011). Massive analysis of rice small RNAs: mechanistic implications of regulated microRNAs and variants for differential target RNA cleavage. Plant Cell 23, 4185-4207. doi: 10.1105/tpc.111.089045

Kansal, S., Mutum, R. D., Balyan, S. C., Arora, M. K., Singh, A. K., Mathur, S., et al. (2015). Unique miRNome during anthesis in drought-tolerant indica rice var. Nagina 22. Planta. 241, 1543-59. doi: 10.1007/s00425-015-2279-3

Katiyar-Agrawal, S., Agarwal, M., and Grover, A. (2003). Heat-tolerant basmati rice engineered by over-expression of hsp101. Plant Mol. Biol. 51, 677-686. doi: 10.1023/A:1022561926676

Kawashima, C. G., Matthewman, C. A., Huang, S., Lee, B. R., Yoshimoto, N., Koprivova, A., et al. (2011). Interplay of SLIM1 and miR395 in the regulation of sulfate assimilation in Arabidopsis. Plant J. 66, 63-76. doi: 10.1111/j.1365-313X.2011.04547.x

Kawashima, C. G., Yoshimoto, N., Maruyama-Nakashita, A., Tsuchiya, Y. N., Saito, K., Takahashi, H., et al. (2009). Sulphur starvation induces the expression of microRNA-395 and one of its target genes but in different cell types. Plant J. 57, 313-321. doi: 10.1111/j.1365-313X.2008.03690.x

Khraiwesh, B., Zhu, J. K., and Zhu, J. (2012). Role of miRNAs and siRNAs in biotic and abiotic stress responses of plants. Biochim. Biophys. Acta 1819, 137-148. doi: 10.1016/j.bbagrm.2011.05.001

Kim, W., Ahn, H. J., Chiou, T. J., and Ahn, J. H. (2011). The role of the miR399-PHO2 module in the regulation of fowering time in response to different ambient temperatures in Arabidopsis thaliana. Mol. Cell 32, 83-88. doi: 10.1007/s10059-011-1043-1

Kruszka, K., Pacak, A., Swida-Barteczka, A., Nuc, P., Alaba, S., Wroblewska, Z., et al. (2014). Transcriptionally and post-transcriptionally regulated microRNAs in heat stress response in barley. J. Exp. Bot. 65, 6123-6135. doi: $10.1093 / \mathrm{jxb} / \mathrm{eru} 353$

Lee, H., Yoo, S. J., Lee, J. H., Kim, W., Yoo, S. K., Fitzgerald, H., et al. (2010). Genetic framework for flowering-time regulation by ambient temperatureresponsive miRNAs in Arabidopsis. Nucleic Acids Res. 38, 3081-3093. doi: $10.1093 / \mathrm{nar} / \mathrm{gkp} 1240$

Lee, S. C., and Luan, S. (2012). ABA signal transduction at the crossroad of biotic and abiotic stress responses. Plant Cell Environ. 35, 53-60. doi: $10.1111 / j .1365-3040.2011 .02426 . x$

Li, H., and Durbin, R. (2009). Fast and accurate short read alignment with Burrows- Wheeler transform. Bioinformatics 25, 1754-1760. doi: 10.1093/bioinformatics/btp324

Li, M. Y., Wang, F., Xu, Z. S., Jiang, Q., Ma, J., Tan, G. F., et al. (2014). High throughput sequencing of two celery varieties small RNAs identifies microRNAs involved in temperature stress response. BMC Genomics 15:242. doi: 10.1186/1471-2164-15-242

Li, Y., Lu, Y. G., Shi, Y., Wu, L., Xu, Y. J., Huang, F., et al. (2014). Multiple rice microRNAs are involved in immunity against the blast fungus Magnaporthe oryzae. Plant Physiol. 164, 1077-1092. doi: 10.1104/pp.113.2 30052

Liu, Q., and Chen, Y. Q. (2010). A new mechanism in plant engineering: the potential roles of microRNAs in molecular breeding for crop improvement. Biotechnol. Adv. 28, 301-307. doi: 10.1016/j.biotechadv.2010.01.002

Lv, D. K., Bai, X., Li, Y., Ding, X. D., Ge, Y., Cai, H., et al. (2010). Profling of cold-stress-responsive miRNAs in rice by microarrays. Gene 459, 39-47. doi: 10.1016/j.gene.2010.03.011

Matsui, T., and Omasa, K. (2002). Rice (Oryza sativa L.) cultivars tolerant to high temperature at flowering: anther characteristics. Ann. Bot. 89, 683-687. doi: $10.1093 / \mathrm{aob} / \mathrm{mcf} 112$

Mayor, A., Martinon, F., De Smedt, T., Pétrilli, V., and Tschopp, J. (2007). A crucial function of SGT1 and HSP90 in inflammasome activity links mammalian and plant innate immune responses. Nat. Immunol. 8, 497-503. doi: 10.1038/ni1459

Moumeni, A., Satoh, K., Kondoh, H., Asano, T., Hosaka, A., Venuprasad, R., et al. (2011). Comparative analysis of root transcriptome profiles of two pairs of drought-tolerant and susceptible rice near-isogenic lines under different drought stress. BMC Plant Biol. 11:174. doi: 10.1186/1471-2229-11-174

Nag, A., and Jack, T. (2010). Sculpting the flower; the role of microRNAs in flower development. Curr. Top. Dev. Biol. 91, 349-378. doi: $10.1016 /$ S0070-2153(10)91012-0
Ni, Z., Hu, Z., Jiang, Q., and Zhang, H. (2013). GmNFYA3, a target gene of miR169, is a positive regulator of plant tolerance to drought stress. Plant Mol. Biol. 82, 113-129. doi: 10.1007/s11103-013-0040-5

Peng, S., Huang, J., Sheehy, J. E., Laza, R. C., Visperas, R. M., Zhong, X., et al. (2004). Rice yields decline with high night temperature from global warming. Proc. Natl. Acad. Sci. U.S.A. 101, 9971 ${ }^{\mathrm{a}}$ 9975. doi: 10.1073/pnas.0403720101

Prasad, P. V. V., Boote, K. J., Allen, L. H. Jr., Sheehy, J. E., and Thomas, J. M. G. (2006). Species, ecotype and cultivar differences in spikelet fertility and harvest index of rice in response to high temperature stress. Field Crops Res. 95, 398-411. doi: 10.1016/j.fcr.2005.04.008

Pressman, E., Shaked, R., and Firon, N. (2007). Tomato response to heat stress: focus on pollen grains. Plant Stress 1, 216-227.

Prus, W., and Filipek, A. (2011). S100A6 mediates nuclear translocation of Sgt1: a heat shock-regulated protein. Amino Acids 41, 781-787. doi: 10.1007/s00726-010-0526-2

Qin, D., Wang, F., Geng, X., Zhang, L., Yao, Y., Ni, Z., et al. (2015). Overexpression of heat stress-responsive TaMBF1c, a wheat (Triticum aestivum L.) multiprotein bridging factor, confers heat tolerance in both yeast and rice. Plant Mol. Biol. 87, 31-45. doi: 10.1007/s11103-014-0259-9

Qin, D., Wu, H., Peng, H., Yao, Y., Ni, Z., Li, Z., et al. (2008). Heat stressresponsive transcriptome analysis in heat susceptible and tolerant wheat (Triticum aestivum L.) by using Wheat Genome Array. BMC Genomics 9:432. doi: 10.1186/1471-2164-9-432

Satake, T., and Yoshida, S. (1978). High temperature induced sterility in indica rices at flowering. Jpn. J. Crop Sci. 47, 6-17. doi: 10.1626/jcs.47.6

Shirasu, K. (2009). The HSP90-SGT1 chaperone complex for NLR immune sensors. Ann. Rev. Plant Biol. 60, 139-164. doi: 10.1146/annurev.arplant.59.032607.092906

Song, J. B., Gao, S., Sun, D., Li, H., Shu, X. X., and Yang, Z. M. (2013). miR394 and LCR are involved in Arabidopsis salt and drought stress responses in an abscisic acid- dependent manner. BMC Plant Biol. 13:210. doi: 10.1186/1471-2229-13-210

Spanudakis, E., and Jackson, S. (2014). The role of microRNAs in the control of flowering time. J. Exp. Bot. 65, 365-380. doi: 10.1093/jxb/ert453

Stief, A., Altmann, S., Hoffmann, K., Pant, B. D., Scheible, W. R., and Bäurle, I. (2014). Arabidopsis miR156 regulates tolerance to recurring environmental stress through SPL transcription factors. Plant Cell 26, 1792-1807. doi: $10.1105 /$ tpc. 114.123851

Sunkar, R., Grike, T., Jain, P. K., and Zhu, J. K. (2005). Cloning and characterization of microRNAs from rice. Plant Cell 17, 1397-1411. doi: 10.1105/tpc.105.031682

Sunkar, R., Kapoor, A., and Zhu, J. K. (2006). Posttranscriptional induction of two cu/zn superoxide dismutase genes in Arabidopsis is mediated by downregulation of miR398 and important for oxidative stress tolerance. Plant Cell 18, 2051-2065. doi: 10.1105/tpc.106.041673

Sunkar, R., and Zhu, J. K. (2004). Novel and stress-regulated microRNAs and other small RNAs from Arabidopsis. Plant Cell 16, 2001-2019. doi: $10.1105 /$ tpc. 104.022830

Tabata, M., Hirabayashi, H., Takeuchi, Y., Ando, I., Iida, Y., and Ohsawa, R. (2007). Mapping of quantitative trait loci for the occurrence of white-back kernels associated with high temperatures during the ripening period of rice (Oryza sativa L.). Breeding Sci. 57, 47-52. doi: 10.1270/jsbbs.57.47

Tian, C., Zuo, Z., and Qiu, J. (2015). Identification and characterization of ABA-responsive microRNAs in rice. J. Genet. Genomics 42, 393-402. doi: 10.1016/j.jgg.2015.04.008

Toki, S., Hara, N., Ono, K., Onodera, H., Tagiri, A., Oka, S., et al. (2006). Early infection of scutellum tissue with Agrobacterium allows high-speed transformation of rice. Plant J. 47, 969-976. doi: 10.1111/j.1365-313X.2006.02836.x

Varkonyi-Gasic, E., Wu, R., Wood, M., Walton, E. F., and Hellens, R. P. (2007). Protocol: a highly sensitive RT-PCR method for detection and quantification of microRNAs. Plant Methods 3:12. doi: 10.1186/1746-4811-3-12

Wang, W., Liu, G., Niu, H., Timko, M. P., and Zhang, H. (2014). The F-box protein $\mathrm{COI}_{1}$ functions upstream of $\mathrm{MYB}_{305}$ to regulate primary carbohydrate metabolism in tobacco (Nicotiana tabacum L. cv. TN ${ }_{90}$ ). J. Exp. Bot. 65, 2147-2160. doi: 10.1093/jxb/eru084

Wu, H. J., Ma, Y. K., Chen, T., Wang, M., and Wang, X. J. (2012). PsRobot: a webbased plant small RNA meta-analysis toolbox. Nucleic Acids Res. 40, W22-W28. doi: $10.1093 /$ nar/gks554 
Wu, M. F., Tian, Q., and Reed, J. W. (2006). Arabidopsis microRNA167 controls patterns of ARF6 and ARF8 expression, and regulates both female and male reproduction. Development 133, 4211-4218. doi: 10.1242/dev.02602

Xia, K., Wang, R., Ou, X., Fang, Z., Tian, C., Duan, J., et al. (2012). OsTIR1 and OsAFB2 downregulation via OsmiR393 overexpression leads to more tillers, early flowering and less tolerance to salt and drought in rice. PLOS ONE 7:e30039. doi: 10.1371/journal.pone.0030039

Xie, F., Wang, Q., Sun, R., and Zhang, B. (2015). Deep sequencing reveals important roles of microRNAs in response to drought and salinity stress in cotton. J. Exp. Bot. 66, 789-804. doi: 10.1093/jxb/eru437

Xin, M., Wang, Y., Yao, Y., Xie, C., Peng, H., Ni, Z., et al. (2010). Diverse set of microRNAs are responsive to powdery mildew infection and heat stress in wheat (Triticum aestivum L.). BMC Plant Biol. 10:123. doi: 10.1186/1471-2229-10-123

Ye, C., Argayoso, M. A., Redona, E., Sierra, S. N., Laza, M. A., Dilla, C. J., et al. (2012). Mapping QTL for heat tolerance at flowering stage in rice using SNP markers. Plant Breeding 131, 33-41. doi: 10.1111/j.1439-0523.2011.0 1924.X

Yi, X., Du, Z., and Su, Z. (2013). PlantGSEA: a gene set enrichment analysis toolkit for plant community. Nucleic Acids Res. 41, W98-W103. doi: $10.1093 /$ nar/gkt281

Young, N. D., and Tanksley, S. D. (1989). Restriction fragment length polymorphism maps and the concept of graphical genotypes. Theor. Appl. Genet. 77, 95-101. doi: 10.1007/BF00292322

Yu, X., Wang, H., Lu, Y., de Ruiter, M., Cariaso, M., Prins, M., et al. (2011). Identification of conserved and novel microRNAs that are responsive to heat stress in Brassica rapa. J. Exp. Bot. 63, 1025-1038. doi: 10.1093/jxb/err337

Zabka, M., Lesniak, W., Prus, W., Kuz Kuźnicki, J., and Filipek, A. (2008). Sgt1 has co-chaperone properties and is up-regulated by heat shock. Biochem. Biophys. Res. Commun. 370, 179-183. doi: 10.1016/j.bbrc.2008.03.055
Zhang, G. Q., Zeng, R. Z., Zhang, Z. M., Ding, X. H., Li, W. T., Liu, G. M., et al. (2004). The construction of a library of single segment substitution lines in rice (Oryza sativa L.). Rice Genet. Newslett. 21, 85-87.

Zhang, T., Zhao, X., Wang, W., Pan, Y., Huang, L., Liu, X., et al. (2012). Comparative transcriptome profiling of chilling stress responsiveness in two contrasting rice genotypes. PLoS ONE 7:e43274. doi: 10.1371/journal.pone.0043274

Zhang, Y. C., Yu, Y., Wang, C. Y., Li, Z. Y., Liu, Q., Xu, J., et al. (2013). Overexpression of microRNA OsmiR397 improves rice yield by increasing grain size and promoting panicle branching. Nat. Biotechnol. 31, 848-852. doi: $10.1038 /$ nbt.2646

Zhou, L., Liu, Y., Liu, Z., Kong, D., Duan, M., and Luo, L. (2010). Genome-wide identification and analysis of drought-responsive microRNAs in Oryza sativa. J. Exp. Bot. 61, 4157-4168. doi: 10.1093/jxb/erq237

Zhou, M., Li, D., Li, Z., Hu, Q., Yang, C., Zhu, L., et al. (2013). Constitutive expression of a miR319 gene alters plant development and enhances salt and drought tolerance in transgenic creeping bentgrass. Plant Physiol. 161, 1375-1391. doi: 10.1104/pp.112.208702

Conflict of Interest Statement: The authors declare that the research was conducted in the absence of any commercial or financial relationships that could be construed as a potential conflict of interest.

Copyright ( 2017 Liu, Yang, Yu, Zhang, Mao, Zhao, Wang, Dong and Liu. This is an open-access article distributed under the terms of the Creative Commons Attribution License (CC BY). The use, distribution or reproduction in other forums is permitted, provided the original author(s) or licensor are credited and that the original publication in this journal is cited, in accordance with accepted academic practice. No use, distribution or reproduction is permitted which does not comply with these terms. 